\title{
Periapical lesions decrease Akt serine phosphorylation and plasma membrane GLUT4 content in rat skeletal muscle
}

\author{
Renato Felipe Pereira ${ }^{1} \cdot$ Max Sander de Oliveira da Mota ${ }^{1}$. \\ Maria Sara de Lima Coutinho Mattera ${ }^{1}$. Thaís Verônica Saori Tsosura ${ }^{1}$. \\ Fernando Yamamoto Chiba $^{2}$ - Cléa Adas Saliba Garbin ${ }^{2} \cdot$ Edilson Ervolino $^{1}$. \\ Luciano Tavares Angelo Cintra ${ }^{3}$ - Maristela Mitiko Okamoto ${ }^{4}$. \\ Ubiratan Fabres Machado ${ }^{4}$. Doris Hissako Sumida ${ }^{1}$
}

Received: 24 April 2015 / Accepted: 11 November 2015 /Published online: 23 November 2015

(C) Springer-Verlag Berlin Heidelberg 2015

\begin{abstract}
Objectives Periapical lesion (PL) promotes insulin resistance; however, the mechanisms underlying this alteration are not fully understood. Therefore, in this study, we aimed to evaluate the Akt serine phosphorylation status and GLUT4 expression levels in the gastrocnemius muscle (GM) of rats with PL. Materials and methods Male Wistar rats $(n=42)$ were distributed equally into control (CN) and PL groups. The pulpal tissue of the PL group rats was exposed to the oral environment for 30 days. Thereafter, glucose and insulin levels were assessed, followed by homeostasis model assessment of insulin resistance (HOMA-IR). The Akt serine phosphorylation and GLUT4 levels of microsomal (M) and plasma membrane (PM) fractions were evaluated by western blotting and analyzed statistically.

Results Compared to $\mathrm{CN}$ group rats, PL group rats had lower insulin sensitivity (as observed by HOMA-IR), lower Akt serine phosphorylation status after insulin stimulus, and lower GLUT4 levels in the PM fraction. However, the M fraction in the PL group did not differ significantly from that of the $\mathrm{CN}$ group.
\end{abstract}

Doris Hissako Sumida

dorishs@foa.unesp.br

1 Programa de Pós-graduação Multicêntrico em Ciências Fisiológicas-SBFis, Department of Basic Sciences, Araçatuba Dental School, Univ Estadual Paulista (UNESP), Rod. Marechal Rondom, km 527/528, Araçatuba, SP 16018-805, Brazil

2 Department of Child and Social Dentistry, Araçatuba Dental School, Univ Estadual Paulista (UNESP), Araçatuba, SP, Brazil

3 Endodontics, Araçatuba Dental School, Univ Estadual Paulista (UNESP), Araçatuba, SP, Brazil

4 Department of Physiology and Biophysics, São Paulo Institute of Biomedical Sciences, University of São Paulo (USP), Av. Prof. Lineu Prestes, 2415, CEP: 05508-900 São Paulo, Brazil
Conclusions PL decreases insulin sensitivity, Akt phosphorylation, and PM GLUT4 content.

Clinical relevance The present study indicates that preventing endodontic disease can thwart insulin resistance.

Keywords Diabetes mellitus $\cdot$ Insulin resistance $\cdot$ Periapical lesions $\cdot$ Glucose transporter type 4

\section{Introduction}

Periapical lesion (PL) can occur as a result of an immune response triggered by the presence of bacteria and toxins in the root canals of teeth. This condition can promote an inflammatory response in the dental pulp and periapical region, resulting in local bone resorption [1].

The putative link between oral inflammation and systemic health is of great interest to the medical and dental community. The existence of a bidirectional relationship between endodontic disease and type 2 diabetes mellitus has been studied [2], and some clinical and experimental studies have shown a high prevalence of PL in humans and animals with uncontrolled diabetes [3,4]. Diabetes mellitus is one of the most common chronic diseases worldwide, and 439 million adults are expected to have diabetes by 2030 [5]. Insulin resistance is a hallmark for type 2 diabetes. Reduced insulin-stimulated glucose transport, which manifests as insulin resistance [6], is associated with chronic low-grade inflammation in obese patients [7]. Moreover, periodontal disease and PL can alter insulin signaling and insulin resistance $[8,9]$. In concordance with these findings, increased pro-inflammatory cytokine levels have been found to correlate with PL and obesity and cause insulin resistance [10-12]. While the exact mechanisms underlying insulin resistance in patients with PL require elucidation, 
changes in the expression levels and translocation of glucose transporter type 4 (GLUT4) in skeletal muscle have been suggested to be crucial for insulin resistance [13].

Insulin stimulates tyrosine phosphorylation of the insulin receptor substrate (IRS)-1 isoform. Subsequently, IRS-1 recruits and activates phosphatidylinositol 3-kinase (PI3K) [14], which activates Akt and then leads to GLUT4 translocation to the plasma membrane. Akt regulates GLUT4 trafficking through its substrate, AS160, a Rab GTPase-activating protein [15]. PL causes insulin resistance and impairs the initial stage of insulin signaling, possibly by reducing tyrosine phosphorylation of pp185 (IRS-1/2), as observed in the adipose tissue of rats with PL [9]. In this study, we hypothesized that PL impairs later steps of insulin signaling and GLUT4 expression. To this end, we determined the levels of glucose and insulin, insulin resistance, and GLUT4 expression in the gastrocnemius muscle (GM) of rats. We believe that the identification of proteins that are dysregulated at various stages of insulin signaling will help in the development of new preventive and therapeutic strategies to ameliorate insulin resistance.

\section{Materials and methods}

\section{Experimental animals}

Forty-two male Wistar rats weighing $250-280 \mathrm{~g}$ were used in this study. The rats were kept in temperature-controlled rooms and were given water and food ad libitum. The local ethics committee approved all experimental protocols according to protocol number 2013/00940.

\section{PL induction}

The rats were randomly distributed into two groups of 21 rats each: the control $(\mathrm{CN})$ group and periapical (PL) group. The PL group rats were anesthetized by an intraperitoneal injection of $87 \mathrm{mg} / \mathrm{kg}$ ketamine (Ketamina Agener; Agener, EmbuGuaçu, SP, Brazil) and $13 \mathrm{mg} / \mathrm{kg}$ xylazine (Dorcipec, Vallée, Montes Claros, MG, Brazil). Pulp chambers of the right upper first molars were accessed using a round bur (Long Neck, Dentsply Maillefer, Petrópolis, RJ, Brazil) connected to high-speed rotation equipment. Exposure to the oral environment for 30 days induced PL in the dental pulp. Then, blood and GM tissues were collected from the anesthetized rats $(50 \mathrm{mg} / \mathrm{kg}$ sodium thiopental; Thiopentax, Cristália Itapira, SP, Brazil) after they were fasted for $14 \mathrm{~h}$. The rats were euthanatized after sample collection.

\section{Glycemia, insulinemia, and HOMA-IR}

Blood was collected in heparinized tubes from ten rats per group. Plasma was isolated by immediate centrifugation at $3000 \times g$ for
15 min at $4{ }^{\circ} \mathrm{C}$ and stored in aliquots at $-80{ }^{\circ} \mathrm{C}$. The plasma samples were used to determine glycemia by the glucose oxidase method (enzymatic glucose; ANALISA Diagnóstica, Belo Horizonte, MG, Brazil) and insulinemia by a radioimmunoassay kit (Sensitive Rat Insulin, SRI-13K, Millipore, St Charles, MO, USA). Insulin resistance was evaluated by the homeostasis model assessment of insulin resistance (HOMA-IR) index, calculated by the formula HOMA-IR = fasting glycemia $(\mathrm{mmol} / \mathrm{L}) \times$ fasting insulinemia $(\mu \mathrm{IU} / \mathrm{mL}) / 22.5[16]$.

\section{Evaluation of Akt serine phosphorylation status in skeletal muscle}

GM was collected from six rats per group before and after the administration of $1.5 \mathrm{U}$ of insulin intravenously over $90 \mathrm{~s}$. Tissue samples were prepared according to a previously described method [17] and subjected to western blotting for quantifying the level of serine phosphorylation using antiphosphoserine AktSer473 antibody (Santa Cruz Biotechnology, CA, USA). $\beta$-Actin antibody (EMD Millipore Corp, Billerica, USA) was the control. Immunoreactive bands were detected by autoradiography using a chemiluminescent substrate kit (GE Healthcare, Buckinghamshire, UK). Quantitative analysis of the blots was performed using Scion Image-Release Beta 3b software (National Institutes of Health, Frederick, MD, USA).

\section{GLUT4 protein analysis}

Tissue samples were homogenized in sucrose buffer, $\mathrm{pH} 7.4$ $(10 \mathrm{mmol} / \mathrm{L}$ Tris-HCl, $1 \mathrm{mmol} / \mathrm{L}$ EDTA, and $250 \mathrm{mmol} / \mathrm{L}$ sucrose), and subjected to differential centrifugations to obtain the plasma membrane (PM)- and microsomal membrane (M)enriched fractions of skeletal muscle [18]. Equal amounts of PM- or M-enriched protein were resolved on a $10 \%$ SDS gel. The blots were quantified by densitometry (ImageQuant TL; Amersham Biosciences UK Limited) and normalized for $\beta$ actin as the internal loading control.

\section{Histological processing of the maxilla}

After the rats were euthanized, their maxillae were dissected, decalcified in $10 \%$ EDTA for 60 days, and processed to generate paraffin-embedded histological sections. Latero-lateral, semi-serial sections $(4-\mu \mathrm{m}$ thick) presented the upper first molar in the longitudinal axis. Hematoxylin and eosin staining of the sections enabled the study of the dental pulp and periapical region.

\section{Statistical analyses}

GraphPad Prism (version 5.0) software was used for statistical analysis. Akt serine phosphorylation was determined by 
Fig. 1 Histological appearance of the periapical region in the control $(\mathrm{CN})$ and periapical lesion (PL) groups. Hematoxylin and eosin staining showed normal appearance of dental pulp and periodontal tissues in the $\mathrm{CN}$ group (a) and the lesion established in the periapical region in the PL group (b). $a b$ alveolar bone, $d p$ dental pulp, $r n$ remnants of pulp necrosis, $p d l$ periodontal ligament, asterisk inflammatory infiltrate. Magnification $\times 250$; scale bars $100 \mu \mathrm{m}$
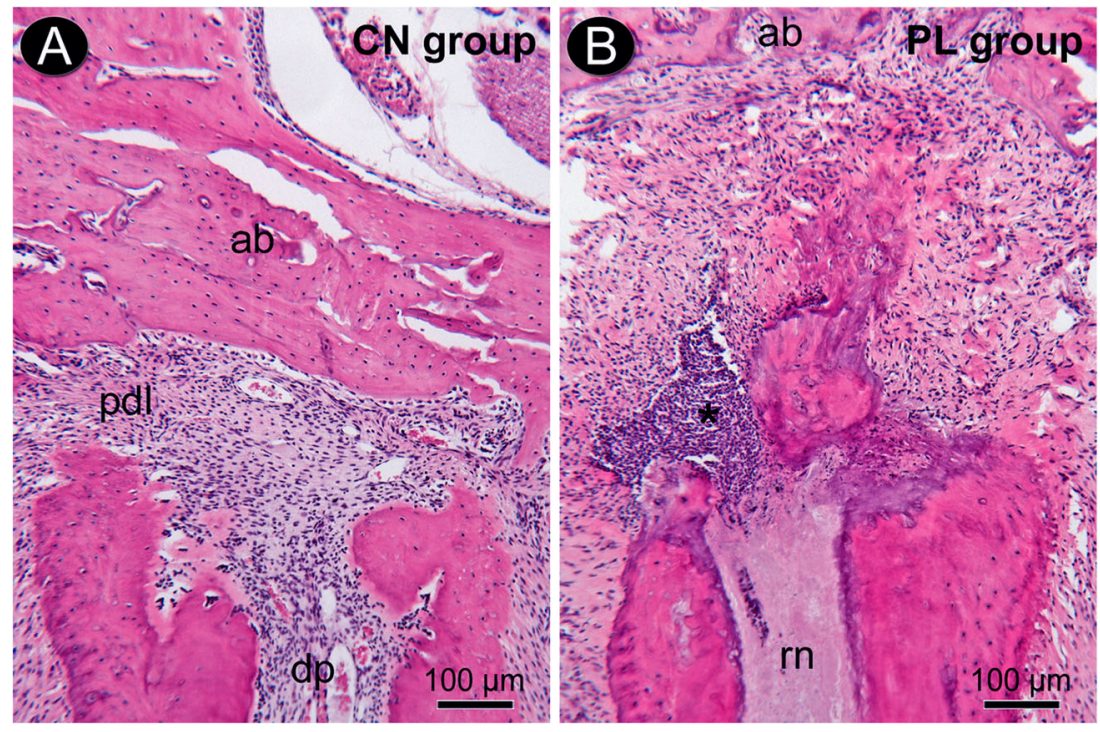

analysis of variance, followed by Tukey's post hoc test. Student's $t$ test was used for the analysis of glycemia, insulinemia, HOMA-IR, and GLUT4 content in M and PM fractions. $P<0.05$ was considered statistically significant.

\section{Results}

\section{Histopathological appearance}

The dental pulp and periodontal tissues in the periapical region of the PL group showed complete necrosis when compared with the dental pulp and periodontal tissues in the periapical region of the $\mathrm{CN}$ group (Fig. 1a). The root canal was either empty or occupied by little necrotic remnants. In addition, the periapical region of every dental root displayed PL of variable sizes. The lesion spanned the alveolar bone in this region, where severe bone resorption had occurred and still showed intense activity (Fig. 1b). The lesions had an inflammatory infiltrate consisting predominantly of lymphocytes and macrophages.

\section{Glycemia, insulinemia, and HOMA-IR}

The glycemia, insulinemia, and HOMA-IR findings are shown in Table 1. The blood glucose and insulin levels were similar in both CN and PL groups. However, the HOMA-IR, calculated from these values, was significantly higher in the PL group than in the $\mathrm{CN}$ group $(P<0.05)$.

\section{Akt serine phosphorylation in the GM}

Compared to the basal levels, insulin stimulus significantly increased Akt serine phosphorylation in both groups in the GM $(P<0.001)$. After insulin stimulus, Akt serine phosphorylation status was significantly lower in the PL group than in the $\mathrm{CN}$ group $(P<0.01$, Fig. 2).

\section{GLUT4 content in the GM}

Figure 3 presents the results of GLUT4 expression in the PM and $\mathrm{M}$ fractions. Both groups had similar GLUT4 expression in the $\mathrm{M}$ fraction; however, compared to the $\mathrm{CN}$ group, the PL group expressed lower levels of GLUT4 in the PM fraction $(P<0.05)$.

\section{Discussion}

In this study, we demonstrated an association between PL and insulin resistance. The reduction in insulin sensitivity in PL rats is in agreement with previous findings of higher insulin resistance and decreased insulin sensitivity in rats with chronic PL $[9,19]$. Because of insulin resistance, plasma insulin and glucose levels might be elevated [18]. However, we did not observe any such elevation in our study. Our results corroborate the findings of Colombo et al. [8], who reported that changes in glycemia in rats with periodontitis were not observed, although these animals were insulin resistant.

Table 1 Mean \pm SEM values of glycemia, insulinemia, and HOMA-IR of $\mathrm{CN}$ and PL groups

\begin{tabular}{lll}
\hline & CN group & PL group \\
\hline Glycemia $(\mathrm{mmol} / \mathrm{L})$ & $7.084 \pm 0.2861$ & $6.783 \pm 0.1211$ \\
Insulinemia $(\mu \mathrm{IU} / \mathrm{mL})$ & $17.38 \pm 5.943$ & $29.47 \pm 5.204$ \\
HOMA-IR & $4.174 \pm 1.479$ & $9.792 \pm 1.564^{*}$ \\
\hline
\end{tabular}

$* P<0.05, \mathrm{CN}$ group vs. PL group 


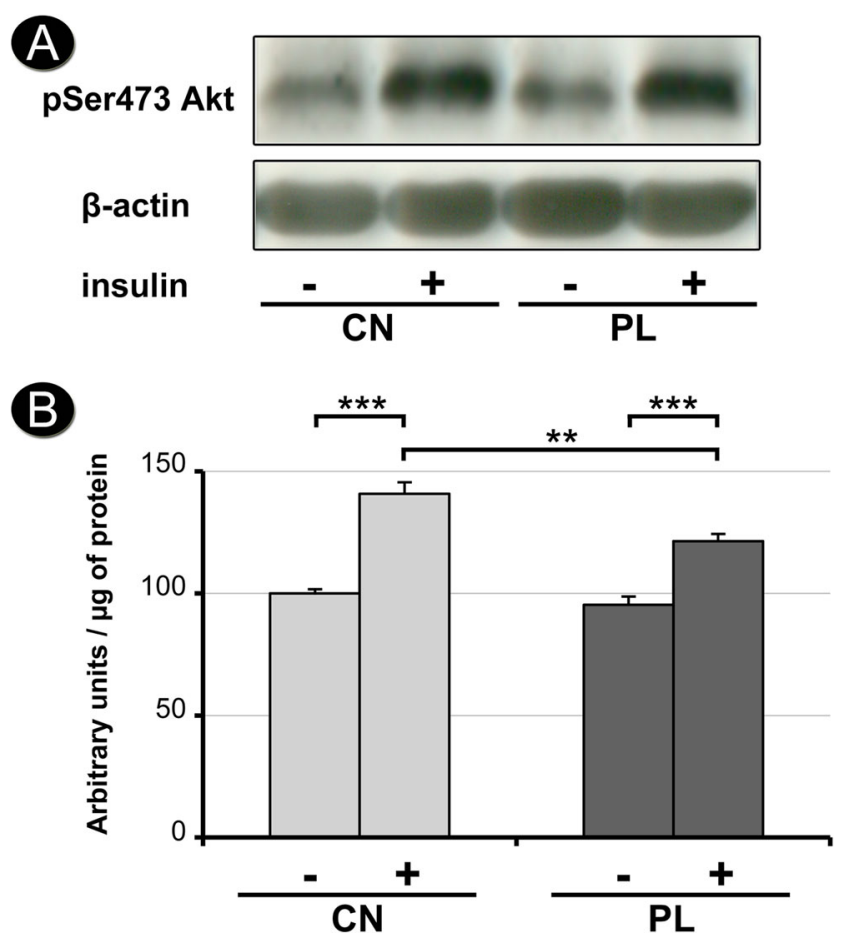

Fig. 2 Evaluation of Akt serine phosphorylation status before (-) and after $(+)$ insulin stimulus in the gastrocnemius muscle (GM). In a, typical autoradiography: equal amounts of protein $(185 \mu \mathrm{g})$ were subjected to SDS-PAGE; $\beta$-actin was used as a control. In $\mathbf{b}$, the values of Akt serine phosphorylation status expressed in arbitrary units are presented as mean \pm SEM $(n=6)$. Asterisks indicate the level of statistical significance

Similarly, Astolphi et al. [9] did not observe changes in glycemia in insulin-resistant rats.

Akt phosphorylation levels indicate the status of insulin signaling, as shown in previous animal studies [20-22]. In the present study, Akt serine phosphorylation reduced after insulin stimulus in the GM of the PL group compared to the $\mathrm{CN}$ group.

Akt phosphorylation also decreases in other insulinsensitive tissues such as adipose tissue. Akt phosphorylation is diminished in 3T3-L1 adipocytes from obese ob/ob mice [23] and in adipocytes infiltrating skeletal muscles in vitro [24]. A periodontitis study associated with a high-fat diet identified decreased Akt activity in rat livers [20]. In these studies, the researchers attributed lower Akt activation to alteration of inflammatory components.

PL triggers inflammation and immune response to combat the invading microorganisms at the dental pulp [25]. A high incidence of macrophages suggests that they are crucial components of periapical granulomas [26]. Macrophage infiltration and activation trigger the production of pro-inflammatory cytokines such as TNF- $\alpha$ [27]. TNF has the capacity to activate the nuclear translocation of transcription factor nuclear factor-kappa B. This factor binds to specific DNA regions and promotes exacerbation of inflammatory processes through the transcription of genes involved in cellular inflammatory and immune responses that are associated with insulin resistance [28]. Studies, including those by our group, have linked TNF- $\alpha$ with impaired insulin signaling. Elevated TNF- $\alpha$ levels inhibit tyrosine phosphorylation of IRS-1, which is associated with obesity [29], periodontitis [8], and PL [9].

Conversely, attenuation of TNF- $\alpha$-induced inflammation has been found to restore tyrosine phosphorylation of IRS-1 and Akt in 3T3-L1 adipocytes [30], suggesting that TNF- $\alpha$ directly affects Akt phosphorylation. Reduced plasma cytokine levels in patients undergoing apicoectomy, curettage [31, 32], tooth extraction [33], or conventional endodontic therapy [34] indicate that treatment of inflammatory disorders such as PL can reverse systemic inflammation. Moreover, inhibiting Akt protein activity decreases GLUT4 translocation to the PM [35].

GLUT4 is a glucose transporter expressed primarily in adipose and muscle tissues [36]. Abnormal GLUT4 trafficking might be the cause of decreased glucose uptake in skeletal muscles in insulin resistance conditions [13]. GLUT4 expression is also reduced in diabetes mellitus and obesity in humans [37] and rodents [38].
Fig. 3 GLUT4 content in the plasma membrane (PM) (a) and microsomal membrane (M) (b). The results are presented as mean \pm SEM $(n=5)$. Asterisks indicate the level of statistical significance

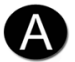

GLUT4 in the PM (plasma membrane)
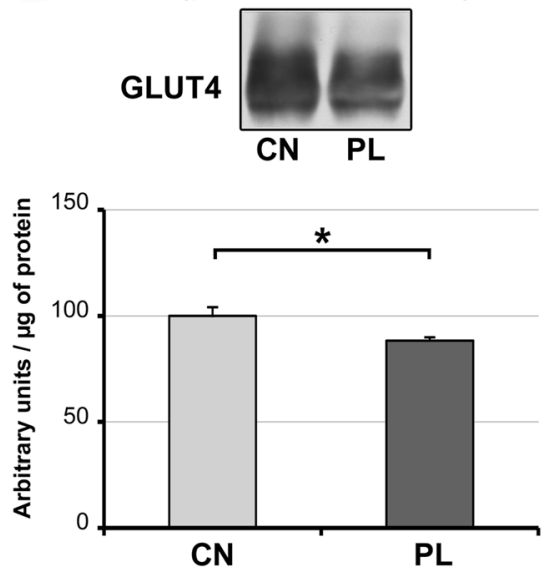

B

GLUT4 in the M (microsomal membrane)

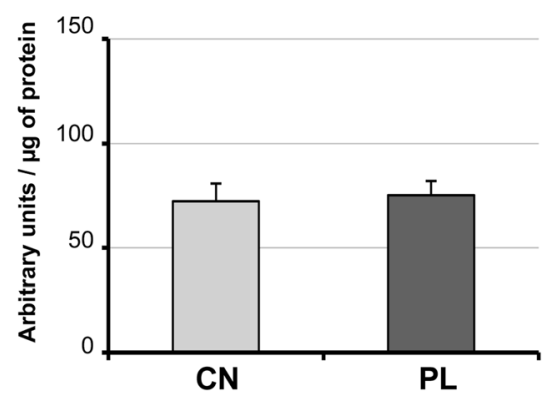


There was no difference in the GLUT4 content in the M fraction in the GM of PL group rats after 30 days of exposure to the illness compared to the $\mathrm{CN}$ group rats. However, reduced GLUT4 levels in the PM fraction in the GM of PL group rats compared to the $\mathrm{CN}$ group rats suggest a reduction in basal glucose uptake. These results corroborate the findings of Carvalho et al. [17], who found difference in GLUT4 content in the GM of obese rats only at 7 months. In yet another study, an experimental model of metabolic syndrome showed decreased GLUT4 content in GM 3 months after disease induction [37]. Thus, the unchanged GLUT4 content in the M fraction in the present study can be attributed to insufficient time for disease progression.

Studies performed with the present experimental model using different exposure times to illness can provide better understanding about the mechanisms underlying the relationship between PL and insulin signaling, enabling the development of more effective therapeutic measures to prevent insulin resistance. Our findings that PL promotes insulin resistance and reduces Akt serine phosphorylation and GLUT4 content in the PM in the GM emphasize the importance of preventing endodontic disease to prevent insulin resistance.

Acknowledgments This study was supported by a grant (2013/14924-0) from the São Paulo Research Foundation (FAPESP), São Paulo, SP, Brazil.

\section{Compliance with ethical standards}

Conflict of interest The authors have no conflicts of interest to declare.

\section{References}

1. Fouad AF, Acosta AW (2001) Periapical lesion progression and cytokine expression in an LPS hyporesponsive model. Int Endod J 34:506-513

2. Segura-Egea JJ, Castellanos-Cosano L, Machuca G et al (2012) Diabetes mellitus, periapical inflammation and endodontic treatment outcome. Med Oral Patol Oral Cir Bucal 17:e356-e361

3. Iwama A, Morimoto T, Tsuji M et al (2006) Increased number of anaerobic bacteria in the infected root canal in type diabetic rats. Oral Surg Oral Med Oral Pathol Oral Radiol Endod 101:681-686

4. Bender IB, Bender AB (2003) Diabetes mellitus and the dental pulp. J Endod 29:383-389

5. Shaw JE, Sicree RA, Zimmet PZ (2010) Global estimates of the prevalence of diabetes for 2010 and 2030. Diabetes Res Clin Pract 87:4-14

6. Kahn BB, Flier JS (2000) Obesity and insulin resistance. J Clin Invest 106:473-481

7. Esser N, Legrand-Poels S, Piette J et al (2014) Inflammation as a link between obesity, metabolic syndrome and type 2 diabetes. Diabetes Res Clin Pract 14:187-189

8. Colombo NH, Shirakashi DJ, Chiba FY et al (2012) Periodontal disease decreases insulin sensitivity and insulin signaling. $\mathrm{J}$ Periodontol 83:864-870
9. Astolphi RD, Curbete MM, Colombo NH et al (2013) Periapical lesions decrease insulin signal and cause insulin resistance. J Endod 39:648-652

10. Zhang J, Gao Z, Yin J et al (2008) S6K directly phosphorylates IRS-1 on Ser-270 to promote insulin resistance in response to TNF-(alpha) signaling through IKK2. J Biol Chem 283:35375353782

11. Coelho M, Oliveira T, Fernandes R (2013) Biochemistry of adipose tissue: an endocrine organ. Arch Med Sci 9:191-200

12. Shoelson SE, Lee J, Goldfine AB (2006) Inflammation and insulin resistance. J Clin Invest 116:2308

13. Garvey WT, Maianu L, Zhu JH et al (1998) Evidence for defects in the trafficking and translocation of GLUT4 glucose transporters in skeletal muscle as a cause of human insulin resistance. J Clin Invest 101:2377-2386

14. Thirone AC, Huang C, Klip A (2006) Tissue-specific roles of IRS proteins in insulin signaling and glucose transport. Trends Endocrinol Metab 17:72-78

15. Thong FS, Dugani CB, Klip A (2005) Turning signals on and off: GLUT4 traffic in the insulin-signaling highway. Physiology (Bethesda) 20:271-284

16. Bonora $\mathrm{E}$, Targher $\mathrm{G}$, Alberiche $\mathrm{M}$ et al (2000) Homeostasis model assessment closely mirrors the glucose clamp technique in the assessment of insulin sensitivity: studies in subjects with various degrees of glucose tolerance and insulin sensitivity. Diabetes Care 23: 57-63

17. Carvalho CR, Brenelli SL, Silva AC et al (1996) Effect of aging on insulin receptor, insulin receptor substrate-1, and phosphatidylinositol 3-kinase in liver and muscle of rats. Endocrinology 137:151-159

18. Mitsumoto Y, Klip A (1992) Developmental regulation of the subcellular distribution and glycosylation of GLUT1 and GLUT4 glucose transporters during myogenesis of L6 muscle cells. J Biol Chem 267:4957-4962

19. Bain JL, Lester SR, Henry WD et al (2009) Effects of induced periapical abscesses on rat pregnancy outcomes. Arch Oral Biol $54: 162-171$

20. Watanabe K, Iizuka T, Adeleke A et al (2011) Involvement of toll like receptor 4 in alveolar bone loss and glucose homeostasis in experimental periodontitis. J Periodontal Res 46:21-30

21. Kristensen JM, Treebak JT, Schjerling P et al (2014) Two weeks of metformin treatment induces AMPK-dependent enhancement of insulin-stimulated glucose uptake in mouse soleus muscle. Am J Physiol Endocrinol Metab 306:1099-1109

22. Melo AM, Benatti RO, Ignacio-Souza LM et al (2014) Hypothalamic endoplasmic reticulum stress and insulin resistance in offspring of mice dams fed high-fat diet during pregnancy and lactation. Metabolism 63:682-692

23. Fan N, Sun H, Wang Y et al (2014) Midkine, a potential link between obesity and insulin resistance. PLoS One 9:e88299

24. Seyoum B, Fite A, Abou-Samra AB (2011) Effects of 3 T3 adipocytes on interleukin-6 expression and insulin signaling in L6 skeletal muscle cells. Biochem Biophys Res Commun 410:13-18

25. Bergenholtz G (1977) Effect of bacterial products on inflammatory reactions in the dental pulp. Scand J Dent Res 85:122-129

26. Marton IJ, Kiss C (1993) Characterization of inflammatory cell infiltrate in dental periapical lesions. Int Endod J 26:131-136

27. Metzger Z (2000) Macrophages in periapical lesions. Endod Dent Traumatol 16:1-8

28. Napetschnig J, Wu H (2013) Molecular basis of NF-kB signaling. Annu Rev Biophys 42:443-468

29. Feinstein R, Kanety H, Papall MZ et al (1993) Tumor necrosis factor-a suppresses insulin-induced tyrosine phosphorylation of insulin receptor and its substrates. J Biol Chem 268:26055-26058

30. Kong P, Chi R, Zhang L et al (2014) Effects of paeoniflorin on tumor necrosis factor- $\alpha$-induced insulin resistance and changes of adipokines in 3T3-L1 adipocytes. Fitoterapia 91:44-50 
31. Márton I, Kiss C, Balla G et al (1988) Acute phase proteins in patients with chronic periapical granuloma before and after surgical treatment. Oral Microbiol Immunol 3:95-96

32. Márton IJ, Kiss C (2000) Protective and destructive immune reactions in apical periodontitis. Oral Microbiol Immunol 15: 139-150

33. Ren YF, Malmstrom HS (2007) Rapid quantitative determination of $\mathrm{C}$-reactive protein at chair side in dental emergency patients. Oral Surg Oral Med Oral Pathol Oral Radiol Endod 104:49-55

34. Kettering JD, Torabinejad M (1984) Cytotoxicity of root canal sealers: a study using HeLa cells and fibroblasts. Int Endod J 17: $60-66$
35. Whiteman EL, Cho H, Birnbaum MJ (2002) Role of Akt/protein kinase B in metabolism. Trends Endocrinol Metab 13:444-451

36. Leto D, Saltiel AR (2012) Regulation of glucose transport by insulin: traffic control of GLUT4. Nat Rev Mol Cell Biol 13:383-396

37. Garvey WT, Maianu L, Huecksteadt TP et al (1991) Pre-translation suppression of a glucose transporter protein causes insulin resistance in adipocytes from patients with non-insulin-dependent diabetes mellitus and obesity. J Clin Invest 87:1072-1081

38. Leguisamo NM, Lehnen AM, Machado UF et al (2012) GLUT4 content decreases along with insulin resistance and high levels of inflammatory markers in rats with metabolic syndrome. Cardiovasc Diabetol 11:100 